\title{
NeuroImage
}

ELSEVIER

www.elsevier.com/locate/ynimg

NeuroImage 31 (2006) $354-365$

\section{Native and non-native reading of sentences: An fMRI experiment}

\author{
Shirley-Ann Rüschemeyer, ${ }^{*}$ Stefan Zysset, and Angela D. Friederici \\ Max-Planck-Institute of Human Cognitive and Brain Sciences, Stephanstr. 1a, Leipzig 04103, Germany
}

Received 28 September 2005; revised 10 November 2005; accepted 18 November 2005

Available online 19 January 2006

\begin{abstract}
The processing of syntactic and semantic information in written sentences by native (L1) and non-native (L2) speakers was investigated in an fMRI experiment. This was done by means of a violation paradigm, in which participants read sentences containing either a syntactic, a semantic, or no violation. The results of this study were compared to those of a previous fMRI study, in which auditory sentence processing in $\mathrm{L} 1$ and $\mathrm{L} 2$ was investigated. The results indicate greater activation for $\mathrm{L} 2$ speakers as compared to $\mathrm{L} 1$ speakers when reading sentences in several language- and motor-related brain regions. The processing of syntactically incorrect sentences elicited no reliably greater activation in language areas in L2 speakers. In L1 speakers, on the other hand, syntactic processing, as compared to semantic processing, was associated with increased activation in left mid to posterior superior temporal gyrus. In response to the processing of semantically incorrect sentences, both $\mathrm{L} 2$ and $\mathrm{L} 1$ speakers demonstrated increased involvement of left inferior frontal gyrus. The results of this study were compared to a previously conducted fMRI study, which made use of identical sentence stimuli in the auditory modality. Results from the two studies are in general agreement with one another, although some differences in the response of brain areas very proximal to primary perceptual processing areas (i.e. primary auditory and visual cortex) were observed in conjunction with presentation in the different modalities. The combined results provide evidence that $L 1$ and L2 speakers rely on the same cortical network to process language, although with a higher level of activation in some regions for L2 processing.

(C) 2005 Elsevier Inc. All rights reserved.
\end{abstract}

\section{Introduction}

How two different languages are represented in the brain remains an open question. Several neuroimaging studies have been conducted investigating the cerebral representation of native (L1) and non-native (L2) language processing, however, the results provide an inconsistent picture. Evidence can be found in the literature supporting distinct cerebral representations of L1 and L2,

\footnotetext{
* Corresponding author. Fax: +49 3419940260.

E-mail address: ruesch@cbs.mpg.de (S.-A. Rüschemeyer).

Available online on ScienceDirect (www.sciencedirect.com).
}

however, as much evidence can be found to support the notion of overlapping cerebral representation of L1 and L2.

Several factors are recognized to contribute to the disagreement between studies (review Abutalebi et al., 2001). First of all, a general delineation must clearly be drawn between L2 production and L2 comprehension studies. Although not all production studies focus on the same cerebral areas, the general conclusion drawn by the vast majority of this research has been that L1 and L2 are represented, at least to some degree, distinctly in the bilingual brain (Kim et al., 1997; Perani et al., 2003). It has been proposed, however, that such differences are related to difficulties in articulating a foreign language, rather than distinct representation of L1 and L2 per se (Klein et al., 1994, 1995). A general limitation of L2 production studies is the fact that researchers have been forced for technical reasons to rely on covert language production, or inner speech. Covert language production is not easily characterized by the experimenter: having participants produce spontaneous inner speech is clearly highly uncontrolled, while using experimental tasks such as covert picture naming, which allow somewhat more control over assumed production, still leaves room for doubt as to what exactly participants are doing.

Studies focusing on L2 comprehension are more heterogeneous in their results than those investigating production. Some researchers have provided evidence for shared cerebral representation of L1 and L2 (Chee et al., 1999b, 2000, 2003; Illes et al., 1999), while others argue for distinct representations of L1 and L2 (Dehaene et al., 1997; Marian et al., 2003; Perani et al., 1996, 1998, Wartenburger et al., 2003). This discrepancy could arise from three main factors differing between studies: (1) differences in the linguistic dimension under investigation (i.e. semantics or syntax); (2) differences in biographical information between L2 participants (i.e. age of L2 acquisition or level of proficiency); (3) differences in modality of presentation (visual or auditory).

Considering the first factor, neurocognitive models of language comprehension point out that various distinct linguistic domains (i.e. syntax, semantics, phonology, prosody) may be supported by distinct portions of a greater language processing network (Friederici, 2002; Ullman, 2004). Proposals regarding the location of regions processing a given level differ, however, the importance of dissociating between syntactic and semantic processing levels is 
evident from the results of both electrophysiological and functional neuroanatomical studies.

This is an important consideration in reviewing the results of L2 studies as the processing of information from different linguistic domains may differ between L1 and L2 speakers differently than the processing of information from another linguistic domain. In other words, while the processing of semantic information might be quite similar between L1 and L2, processing of syntactic structure may be quite different. In fact, precisely, this distinction between semantic and syntactic processing has been suggested (for review of evidence for shared lexical storage system, see French and Jacquet, 2004). If semantic information in L1 and L2 is supported by the same cortical network, but syntactic information is not, this will clearly cause studies conducted at the word level (Klein, 2003; Chee et al., 1999b, 2000, 2003) to show greater similarities between L1 and L2 than studies conducted at the sentence or discourse level (Dehaene et al., 1997; Perani et al., 1996, 1998; Wartenburger et al., 2003).

The second factor to be considered in investigations of L2 language processing concerns biographical details of the L2 participants under investigation (review Perani and Abutalebi, 2005). It has been shown in behavioral, electrophysiological, and neuroimaging studies that age of L2 acquisition, proficiency level in L2, and level of exposure to L2 can greatly influence how an L2 is processed (Tan et al., 2003; Perani et al., 1996, 1998; Wartenburger et al., 2003; Weber-Fox and Neville, 1996).

The third factor to be considered in L2 comprehension studies is that of modality. The results of studies addressing L2 processing in the visual and auditory modalities are often addressed together. This pooling of evidence may, however, not be justified. A direct contrast between the processing of L2 language stimuli presented in different modalities has, to our knowledge, not been undertaken, however, comparisons have been made between the processing of auditory vs. visual language stimuli for L1 speakers alone (Chee et al., 1999a; Michael et al., 2001). In these studies, small but stable differences between language processing in different modalities are reported.

The fact that the modality of language presentation affects the manner in which language is processed in L1 speakers makes the comparison of results of L2 studies in different modalities rather difficult. In fact, those studies that argue for common representation of L1 and L2 are frequently based on visually presented experimental materials (Chee et al., 1999b, 2000, 2003), while studies reporting differences have been conducted using auditory language stimuli (Dehaene et al., 1997; Perani et al., 1996, 1998). Much of the work in the visual domain has been done with Chinese-English bilinguals, capitalizing on the very different orthographic systems underlying Chinese and English. While Chee and colleagues come to the conclusion that the cerebral representations of L1 and L2 overlap in the bilingual brain, a second proposal suggests that the overlapping results are the result of the modality of investigation (i.e. language reading). Specifically, it is suggested that neural networks supporting reading do differ between monolingual speakers of two different languages but that, in bilingual speakers, neural mechanisms supporting reading in L1 are implemented in reading L2 (Tan et al., 2003; Luke et al., 2002).

Each of the factors introduced here can influence the representation of L2 processing alone, however, interactions between the factors must also be taken into consideration. For example, Wartenburger and colleagues investigated to what degree age of L2 acquisition and proficiency level (Factor 2) influenced the processing of both syntactic and semantic information (Factor 1)
(Wartenburger et al., 2003). They found that, while age of L2 acquisition affected the neural representation of syntactic processing, it had little effect on the representation of semantic processing. Proficiency level in an L2, on the other hand, did influence the neural representation of semantic information in $\mathrm{L} 2$.

In the current study, we focused on the possible interaction between modality type (Factor 3 ) and the processing of information on different linguistic levels (Factor 1) while keeping L2 proficiency constant. To this end, we presented L1 and highly proficient L2 (late learners) of German with written sentences, some of which contained violations of either a syntactic or semantic nature. Changes in the hemodynamic response of the different participant groups correlated with violations in each linguistic domain were compared. The results obtained from this experiment were subsequently compared to the results of a previously published study, in which we presented exactly the same sentence materials to L1 and L2 speakers in the auditory rather than the visual modality (Rüschemeyer et al., 2005). In this manner, we contribute both to research regarding the processing of information from different linguistic domains (syntax and semantics) in sentences, as well as provide a comparison of processes between modalities (visual and auditory).

For the processing of syntactic and semantic information in sentences, we expected to see a similar pattern of activation to that which we observed in response to spoken sentences. Specifically, in response to the detection of a syntactic violation, we expected to see increased involvement of left superior temporal gyrus (STG). Previous literature has suggested that anterior portions of STG may be important in the processing of morphosyntactic and phrase structure information in spoken sentences (Dronkers et al., 2004; Friederici et al., 2003; Rüschemeyer et al., 2005). For the processing of semantically violated sentences, we predicted selectively increased involvement of left inferior frontal gyrus (IFG). This prediction again finds support in the results of previous studies investigating processing of semantic anomalies (Kiehl et al., 2002; Newmann et al., 2001; Rüschemeyer et al., 2005). Differences between the L1 and L2 participants were predicted in response to detection of syntactic violations, but not necessarily in response to semantic violations. This pattern of activation between groups was observed in our previous acoustically presented study (Rüschemeyer et al., 2005), as well as in a number of electrophysiological studies (Hahne, 2001; review see Mueller, 2005).

Previous literature suggests that, while native speakers of two different native languages may process written language in their respective L1s differently from one another, a single speaker having mastered two languages is apt to apply $\mathrm{L} 1$ reading techniques to the reading of L2 as well (Tan et al., 2003). Therefore, we expected to see substantial differences between L1 and L2 groups reading German sentence materials. In particular, we expected to see more involvement in L2 than L1 participants in areas supporting grapheme to phoneme conversion. Our comparison with the earlier auditory study will reveal to what extent processing of written and auditory sentence material rely on similar or different brain structures.

\section{Materials and methods}

\section{Sentence materials}

The experimental material consisted of short sentences using transitive verbs in the imperfect passive form. Participle forms of 
96 different transitive verbs, all of which started with the regular German participle morpheme 'ge', were used to create the experimental sentences. For each participle, three different critical sentences (correct, syntactically incorrect, and semantically incorrect) and one filler sentence were constructed.

Correct sentences were short sentences in the passive voice, consisting of a noun phrase (NP) followed by an auxiliary verb and a sentence-final verb participle. Syntactic anomalies were created by initiating a prepositional phrase through one of seven prepositions (in, zu, unter, vor, am bei, für), which requires completion of phrase structure by a noun phrase (NP). Instead of the requisite NP, the preposition was followed by a verb participle, which cannot be integrated into the established phrase structure and which therefore constitutes a phrase structure violation on the basis of word category. Semantic anomalies were created by presenting a sentence-final verb participle that could not be integrated into the established sentence context on a conceptual level. Such sentences had intact phrase structure (that is, they were syntactically correct). Filler sentences served two functions in this experiment: (1) they were presented in order to balance the number of correct and incorrect sentence materials in the experiment (all filler sentences were syntactically and semantically correct) and (2) they prevented participants from being able to detect a phrase structure violation based solely on the presence of a preposition.

Two differently randomized stimulus sequences were designed for the experiment. The 96 sentences from each of the four conditions were systematically distributed between two lists, so that each verb occurred in only two out of four conditions in the same list. The lists were then pseudo-randomized with the constraints that (a) repetitions of the same participle were separated by at least 20 intervening trials, (b) no more than three consecutive sentences belonged to the same condition and (c) no more than four consecutive trials contained either correct or incorrect sentences. Furthermore, the regularity with which the two conditions followed one another was matched for all combinations. The order of stimuli in each of the two randomized stimulus sequences was then reversed, yielding four different lists. These were distributed randomly across participants.

An experimental session consisted of three 11-min blocks. Blocks consisted of an equal number of trials and a matched number of items from each condition. Each session contained 240 trials, consisting of 144 critical trials ( $48 \times$ each critical condition) plus 48 filler trials and 48 null events, in which no stimulus was presented and the BOLD response was allowed to return to a baseline state.

All trials lasted $8 \mathrm{~s}$ (i.e. 4 scans at $\mathrm{TR}=2 \mathrm{~s}$ ). The onset of each stimulus presentation relative to the beginning of the first of the four scans was randomly varied $(0,500,1500,2000 \mathrm{~ms})$ in order to enhance the temporal resolution of the signal captured. Participants were unaware of this modulation. Each trial was introduced by a fixation cross, which was presented in the center of the screen for $400 \mathrm{~ms}$. Following this, sentences were presented visually in a word-by-word fashion. The first noun phrase was presented as a whole (meaning determiner and noun were presented together); all subsequent words were presented in isolation. Each word was presented for $500 \mathrm{~ms}$ in the center of the screen with no interstimulus interval. The length of sentence presentation therefore clearly differed between the different conditions, depending on the number of words presented (COR $=1500 \mathrm{~ms}, \mathrm{SYN}=2000 \mathrm{~ms}$, $\mathrm{SEM}=1500 \mathrm{~ms}$, FILLER $=2500 \mathrm{~ms}$ ). After presentation of the final word for $500 \mathrm{~ms}$, participants were cued by three centrally presented question marks to judge the acceptability of the sentence. Sentence acceptability was judged by participants via button press. Participants held a response box in their right hand and were requested to push one of two buttons depending on the grammaticality of each sentence. Participants were allowed $2 \mathrm{~s}$ to respond to sentences. Performance rates and reaction times were recorded.

Null events were also introduced by an initial fixation cross. Following the fixation cross, subjects saw only a blank screen for the duration of the trial (Table 1).

\section{Participants}

Eighteen native speakers of German ( 9 females), age 22-34 (mean age 26.2, $\mathrm{SD}=3$ ), and 16 non-native speakers of German (native speakers of Russian; 13 females), age 18-36 (mean age 26, $\mathrm{SD}=5.4$ ), participated in this experiment after giving informed written consent.

Non-native participants were native speakers of Russian, who had been residing in Germany for 8 years on average and had been speaking German for 7 years $($ mean $=7$; SD $=4.3$ ). They were highly proficient in German, had generally attended a German high school, but spoke Russian at home and with their families. Participants were asked to fill out a questionnaire regarding their language abilities prior to the experimental session, which was used to pre-select participants to some degree. Participants were asked, for example, to judge their own proficiency in reading and writing in German on a 10-point scale $(1=$ very difficult to read the newspaper, $10=$ very easy to read the newspaper; $1=$ very difficult to express ideas in writing, $10=$ very easy to express ideas in writing) as well as to estimate what percentage of their total reading time was spent with literature in German. Participants included in the experiment rated their written verbal skills to be quite high (reading: mean $=7.6 ; \mathrm{SD}=1.7$; writing: mean $=7.7 ; \mathrm{SD}=1.6$ ) and read approximately $75 \%$ (mean $=75 ; \mathrm{SD}=17.3)$ of the time in German. Nevertheless, the final test of proficiency remained performance in the actual scanning environment, and two participants who did not perform within the realms $(2.5 * \mathrm{SD})$ of the rest of the group were excluded from further analysis.

\section{fMRI data acquisition}

Participants were placed in the scanner in a supine position. Sentences were presented on a computer screen outside of the scanner, which participants could see via mirror glasses.

Twenty axial slices ( $4 \mathrm{~mm}$ thickness, $1 \mathrm{~mm}$ inter-slice distance, FOV $19.2 \mathrm{~cm}$, data matrix of $64 \times 64$ voxels, in-plane resolution of $3 \times 3 \mathrm{~mm}$ ) were acquired every $2 \mathrm{~s}$ during function measurements

Table 1

Sentence materials

\begin{tabular}{ll}
\hline Condition & Example in German with English translation equivalent \\
\hline COR & $\begin{array}{l}\text { Das Brot wurde gegessen. } \\
\text { The bread was eaten }\end{array}$ \\
SYN & $\begin{array}{l}\text { Das Eis wurde im gegessen. } \\
\text { The ice-cream was in-the eaten }\end{array}$ \\
SEM & $\begin{array}{l}\text { Der Vulkan wurde gegessen. } \\
\text { The volcano was eaten }\end{array}$ \\
FILLER & Die Pizza wurde im Restaurant gegessen. \\
& The pizza was in-the restaurant eaten
\end{tabular}

Examples of sentence stimuli. 
(BOLD sensitive gradient EPI sequence, $\mathrm{TR}=2 \mathrm{~s}, \mathrm{TE}=30 \mathrm{~ms}$, flip angle $=90$, acquisition bandwidth $=100 \mathrm{~Hz}$ ) with a $3 \mathrm{~T}$ Bruker Medspec 30/100 system. Prior to functional imaging, T1-weighted MDEFT images (data matrix $256 \times 256$, TR $1.3 \mathrm{~s}$, TE $10 \mathrm{~ms}$ ) were obtained with a non-slice-selective inversion pulse followed by a single excitation of each slice (Norris, 2000). These images were used to co-register functional scans with previously obtained highresolution whole head 3D brain scans-128 sagittal slices, $1.5 \mathrm{~mm}$ thickness, FOV $25.0 \times 25.0 \times 19.2 \mathrm{~cm}$, data matrix of $256 \times 156$ voxels (Lee et al., 1995).

\section{fMRI data analysis}

The functional imaging data were processed using the software package LIPSIA (Lohmann et al., 2001). Functional data were corrected first for motion artifacts using a matching metric based on linear correlation. Data were subsequently corrected for the temporal offset between slices acquired in one scan using a cubicspline interpolation based on the Nyquist-Shannon Theorem. Low-frequency signal changes and baseline drifts were removed by applying a temporal highpass filter to remove frequencies lower than $1 / 80 \mathrm{~Hz}$, and a spatial Gaussian filter with $5.65 \mathrm{~mm}$ FWHM was applied.

To align the functional dataslices with a $3 \mathrm{D}$ stereotactic coordinate reference system, a rigid linear registration with six degrees of freedom (3 rotational, 3 translational) was performed. The rotational and translational parameters were acquired on the basis of the MDEFT (Norris, 2000) and EPI-T1 slices to achieve an optimal match between these slices and the individual 3D reference data set, which was acquired during a previous scanning session. The MDEFT volume data set with 160 slices and $1 \mathrm{~mm}$ slice thickness was standardized to the Talairach stereotactic space. The rotational and translational parameters were subsequently transformed by linear scaling to a standard size. The resulting parameters were then used to transform the functional slices using trilinear interpolation, so that the resulting functional slices were aligned with the stereotactic coordinate system. This linear normalization process was improved by a subsequent processing step that performed an additional nonlinear normalization (Thiron, 1998). The transformation parameters obtained from both normalization steps were subsequently applied to the functional data. Voxel size was interpolated during co-registration from $3 \times 3 \times 4$ $\mathrm{mm}$ to $3 \times 3 \times 3 \mathrm{~mm}$.

The statistical evaluation was based on a least-squares estimation using the general linear model for serially autocorrelated observations (Worsley and Friston, 1995). The design matrix was generated with a synthetic hemodynamic response function and its first and second derivatives (Friston et al., 1998; Josephs et al., 1997). The model equation, made up of the observed data, the design matrix, and the error term, was convolved with a Gaussian kernel of dispersion of $4 \mathrm{~s}$ FWHM.

\section{Within group contrast}

For each participant, four contrast images were generated. Three of these contrasts were used in the subsequent contrast between groups. These represented the main effects of processing (1) correct, (2) syntactically incorrect, and (3) semantically incorrect sentences vs. null events. The fourth contrast created was interesting for the within group analysis. Here, we calculated the main effects of syntactically violated sentences vs. semantically violated sentences. Because individual functional data sets had been aligned to the standard stereotactic reference space, a group analysis based on the contrast images could be performed. Singleparticipant contrast images were entered into a second-level random effects analysis for each of the contrasts. The group analysis consisted of a one-sample $t$ test cross the contrast images of all subjects that indicated whether observed differences between conditions were significantly distinct from zero. Subsequently, $t$ values were transformed into $Z$ scores. To protect against false positive activation, a double threshold was applied, by which only regions with a $Z$ score exceeding 3.09 ( $P<0.001$, uncorrected) and a volume exceeding 14 voxels $\left(378 \mathrm{~mm}^{3}\right)$ were considered (Forman et al., 1995). This is equivalent to a significance level of $P<0.05$ (corrected). Within the greater regions of activation, local maxima were determined, which had a significance level of $P<$ 0.001 (uncorrected) at the voxel level and which were the highest point of activation within a volume of 14 connected voxels.

\section{Between group contrast}

The between group analysis consisted of a two-sample $t$ test of the contrast images (correct-null, semantic violation-null, syntactic violation-null) between the two groups (L1, L2). The contrast images were compared voxelwise to determine whether observed differences between the groups differed significantly from zero. $t$ values were transformed into $Z$ scores. To protect against false positive activation, a double threshold was again applied, by which only regions with a $Z$ score exceeding $3.09(P<0.001$, uncorrected) and a volume exceeding 14 voxels $\left(378 \mathrm{~mm}^{3}\right)$ were considered.

\section{Results}

\section{Behavioral results}

Accuracy of responses and reaction times were recorded during the functional measurement. Reaction times are, however, not reported as participants were prompted to make a delayed judgment only after the sentence had been presented in full.

Non-native speakers showed in their performance that they were capable of carrying out the task and of correctly judging most sentences (see Fig. 1). Differences in the performance of participants were analyzed in a repeated measures ANOVA with the dependent variable performance rate and the independent variables Group (L1, L2) and Condition (COR, SEM, SYN). The

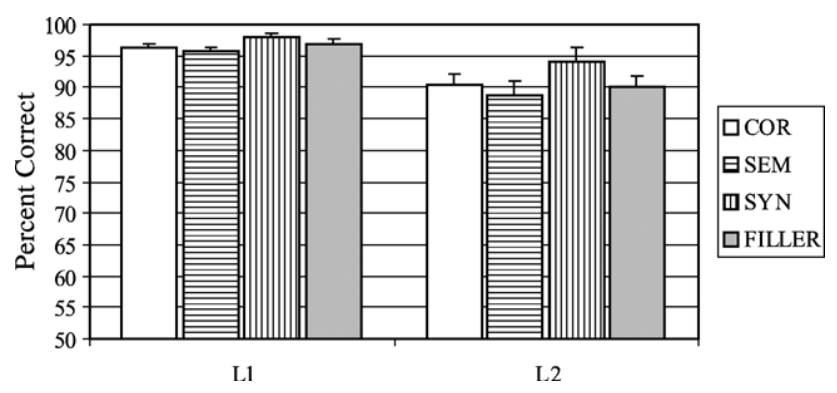

Fig. 1. Performance rates (percent correct) for native (L1) and non-native (L2) speakers in response to correct (COR), syntactically incorrect (SYN), semantically incorrect (SEM), and non-critical (FILLER) sentence stimuli. 
performance rate of filler sentences was not included in the statistical analysis in order to maintain consistency between the behavioral and neuroimaging data reported. Nevertheless, performance rates for this condition can be seen in Fig. 1. Despite the high performance of non-native speakers in all conditions, L2 participants were not as proficient at judging sentences as native speakers, as indicated by a main effect of Group $[F(1,32)=14$, $P<0.001]$. Additionally, a main effect of Condition was observed $[F(2,64)=4, P<0.05]$. No Group $\times$ Condition interaction was observed.

\section{Imaging results}

\section{Between group: native vs. non-native speakers}

The direct comparison of activation elicited by each experimental condition between native and non-native participants showed no regions of reliably greater activation for native speakers for any condition (see Table 2, Fig. 2).

In response to correct sentences, non-native speakers showed greater activation than native speakers in several motor-related areas including the pre-supplementary motor area (preSMA), the bilateral precentral gyri, as well as bilateral regions within central sulcus extending into postcentral gyrus and the angular gyrus (in the left hemisphere). Additionally, increased activation of bilateral structures in the basal ganglia (caudate nucleus) was observed. Non-native speakers demonstrated increased levels of activation within several non-motor-related cortices as well, including left ventrolateral prefrontal cortex, extending from the left inferior frontal sulcus (IFS) into the inferior frontal gyrus (IFG; pars opercularis, pars triangularis). In the right hemisphere, prefrontal activation was far reduced, encompassing only the right frontal operculum. Increased activation was also observed for non-native as compared to native speakers in the left middle temporal gyrus (MTG).
In response to syntactically anomalous sentences, non-native speakers showed greater levels of activation than native speakers in preSMA, right precentral gyrus, right frontal operculum, and structures of the bilateral basal ganglia.

In response to semantically anomalous sentences, non-native speakers showed greater levels of activation than native speakers in preSMA, right precentral gyrus, right frontal operculum, bilateral postcentral sulcus, the right lingual gyrus, and the bilateral structures of the basal ganglia. Additionally, increased activation was observed in the left anterior superior temporal gyrus (STG).

\section{Within group: native speakers}

Native speakers showed reliably more activation in correlation with syntactically anomalous than semantically anomalous sentences in right inferior precentral sulcus, bilateral intraparietal sulcus, extending in the left hemisphere towards medial regions in the precuneus and in the right hemisphere to lateral aspects of the supramarginal gyrus (see Table 3, Fig. 3). Additionally, selective activation was observed in left STG, extending from posterior superior temporal sulcus (STS) to mid-STG and a region in the STG anterior to Heschl's gyrus. In the right hemisphere, activation was observed in the IFG. Increased activation for the processing of syntactic anomalies was also observed in the bilateral lateral occipital cortex.

For the processing of semantic anomalies, increased activation was observed in native speakers in the left anterior IFG.

\section{Within group: non-native speakers}

Non-native speakers showed increased activation for the processing of syntactic anomalies vs. semantic anomalies in the right intraparietal sulcus, extending to both medial aspects in the precuneus and lateral supramarginal gyrus (Table 3, Fig. 3).

Table 2

Direct contrasts between native (L1) and non-native (L2) groups for each sentence condition

\begin{tabular}{|c|c|c|c|c|c|c|c|c|c|c|c|c|c|c|c|}
\hline \multirow[t]{2}{*}{ Region } & \multicolumn{5}{|c|}{ Correct } & \multicolumn{5}{|c|}{ Syntactic violation } & \multicolumn{5}{|c|}{ Semantic violation } \\
\hline & $x$ & $y$ & $z$ & $Z \max$ & Volume & $x$ & $y$ & $z$ & $Z \max$ & Volume & $x$ & $y$ & $z$ & $Z \max$ & Volume \\
\hline Pre-supplementary motor area & -7 & 11 & 59 & 4.6 & 2698 & -8 & 11 & 56 & 3.7 & 3535 & -10 & 11 & 53 & 4 & 3313 \\
\hline Left precentral gyrus & -31 & -7 & 56 & 2.9 & 1176 & & & & & & & & & & \\
\hline Right precentral gyrus & 32 & 2 & 53 & 3.3 & 971 & 32 & 5 & 53 & 3.6 & 1526 & 28 & -1 & 56 & 3.8 & 6210 \\
\hline Left inferior frontal sulcus & -49 & 2 & 32 & 3.2 & 711 & & & & & & & & & & \\
\hline Left inferior frontal gyrus (BA44) & -50 & 14 & 18 & 3.1 & 613 & & & & & & & & & & \\
\hline Left inferior frontal gyrus (BA45/47) & -50 & 26 & 6 & 3.3 & 952 & & & & & & & & & & \\
\hline Right frontal operculum & 35 & 23 & 6 & 3.9 & 816 & 37 & 23 & 3 & 3.9 & 879 & 37 & 23 & 6 & 3.8 & 599 \\
\hline Left central sulcus-postcentral gyrus & -53 & -22 & 47 & 3.6 & 823 & -52 & -19 & 50 & 3.7 & 3764 & & & & & \\
\hline $\begin{array}{l}\text { Left inferior postcentral sulcus- } \\
\text { supramarginal gyrus }\end{array}$ & -41 & -28 & 38 & 3.2 & 411 & -38 & -31 & 41 & 3.3 & 731 & -41 & -28 & 38 & 4.1 & 11,985 \\
\hline Left angular gyrus & -31 & -43 & 47 & 3.5 & 1347 & -31 & -43 & 47 & 3 & 861 & & & & & \\
\hline Right postcentral gyrus & 35 & -28 & 41 & 3.5 & 4697 & 37 & -28 & 41 & 3.6 & 4916 & & & & & \\
\hline Right superior postcentral sulcus & & & & & & & & & & & 26 & -58 & 53 & 3.6 & 903 \\
\hline Left anterior superior temporal gyrus & & & & & & & & & & & -56 & -7 & -3 & 3.2 & 533 \\
\hline Left middle temporal gyrus & -64 & -46 & 3 & 3.8 & 1196 & & & & & & & & & & \\
\hline Right lingual gyrus & & & & & & & & & & & 7 & -58 & 3 & 3.6 & 3454 \\
\hline Left basal ganglia & -11 & 17 & 12 & 3.2 & 510 & -14 & 17 & 12 & 3.2 & 483 & -14 & 17 & 12 & 3.8 & 2404 \\
\hline Right basal ganglia & 8 & 11 & 15 & 3.1 & 235 & 8 & 11 & 15 & 3 & 182 & 10 & 11 & 15 & 3.4 & 1167 \\
\hline
\end{tabular}

Regions of significantly different activation between groups $(P<0.05$, corrected). Non-native (L2) speakers showed greater activation than native (L1) speakers in the listed regions. L1 speakers showed no regions to be activated to a greater degree than L2 speakers. Listed are the location, Talairach coordinates, maximum $Z$ values, and cluster extents $\left(\mathrm{mm}^{3}\right)$ for each identified activation $(\mathrm{L} 2>\mathrm{L} 1)$ in response to correct, syntactically violated, and semantically violated sentences. 

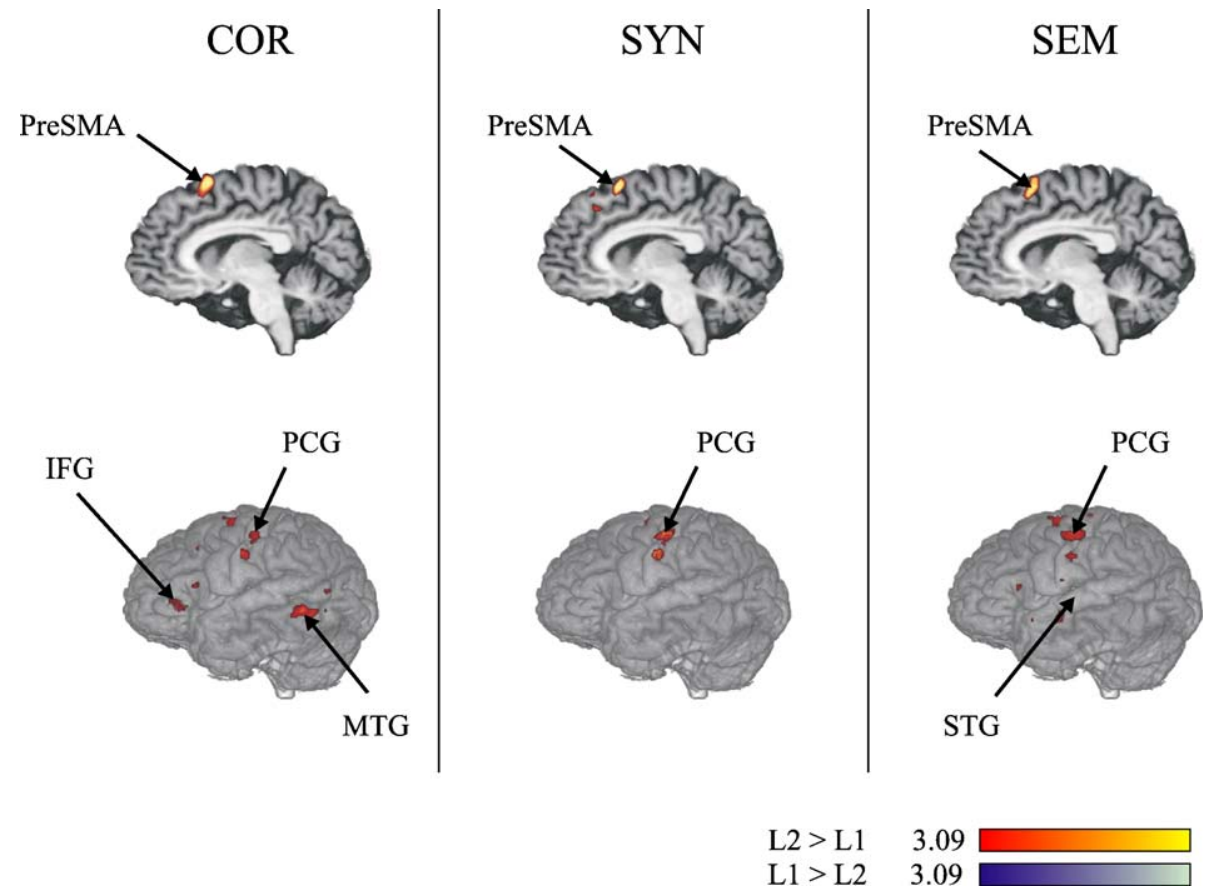

Fig. 2. Direct contrasts between native (L1) and non-native (L2) groups $(Z>3.09)$. Areas showing more activation for L2 speakers are depicted in red; areas showing more activation for L1 speakers are depicted in blue. L2 speakers show increased levels of activation in pre-supplementary motor area (preSMA), left inferior frontal gyrus (IFG), pre- and postcentral gyrus (PCG), as well as middle and superior temporal gyrus (MTG, STG). L1 speakers demonstrated no regions to be more highly activated than for $\mathrm{L} 2$ speakers.

Increased activation was observed additionally in the right lateral occipital cortex.

In response to semantic violations, non-native speakers showed reliably greater level of activation in the left anterior IFG. This activation had 2 local maxima, one located within deep frontal operculum, approaching anterior insular cortex, and one on the lateral surface of the pars triangularis.

\section{Discussion}

Differences in the processing of a native vs. non-native language were captured in the direct contrast of brain activation between participant groups (L1 vs. L2) during processing of sentences in each experimental condition. In response to sentence stimuli in all experimental conditions, L1 speakers showed no

Table 3

Within group contrasts between sentence conditions

\begin{tabular}{|c|c|c|c|c|c|c|c|c|c|c|c|}
\hline Brain region & $x$ & $y$ & $z$ & $\begin{array}{l}\text { Cluster } \\
\text { extent }\end{array}$ & $Z \max$ & Brain region & $x$ & $y$ & $z$ & $\begin{array}{l}\text { Cluster } \\
\text { extent }\end{array}$ & $Z \max$ \\
\hline Native speakers & & & & & & Non-native speakers & & & & & \\
\hline $\mathrm{SYN}>\mathrm{SEM}$ & & & & & & $\mathrm{SYN}>\mathrm{SEM}$ & & & & & \\
\hline Right inferior precentral sulcus & 46 & 3 & 21 & 40 & 4.01 & & & & & & \\
\hline Left intraparietal sulcus-precuneus & $\begin{array}{l}-32 \\
-10\end{array}$ & $\begin{array}{l}-57 \\
-68\end{array}$ & $\begin{array}{l}50 \\
50\end{array}$ & 204 & $\begin{array}{l}4.59 \\
3.85\end{array}$ & $\begin{array}{l}\text { Right intraparietal sulcus- } \\
\text { precuneus }\end{array}$ & $\begin{array}{l}10 \\
25\end{array}$ & $\begin{array}{l}-69 \\
-65\end{array}$ & $\begin{array}{l}44 \\
44\end{array}$ & 104 & $\begin{array}{l}4.23 \\
4.01\end{array}$ \\
\hline $\begin{array}{l}\text { Right intraparietal sulcus- } \\
\text { supramarginal gyrus }\end{array}$ & $\begin{array}{l}55 \\
34\end{array}$ & $\begin{array}{l}-42 \\
-50\end{array}$ & $\begin{array}{l}29 \\
41\end{array}$ & 384 & $\begin{array}{l}4.8 \\
4.51\end{array}$ & $\begin{array}{l}\text { Right intraparietal sulcus- } \\
\text { supramarginal gyrus }\end{array}$ & $\begin{array}{l}54 \\
43\end{array}$ & $\begin{array}{l}-36 \\
-35\end{array}$ & $\begin{array}{l}44 \\
44\end{array}$ & 247 & $\begin{array}{l}4.7 \\
4.69\end{array}$ \\
\hline Left posterior superior temporal sulcus- & -49 & -45 & 13 & 208 & 5.09 & & & & & & \\
\hline & $\begin{array}{l}-58 \\
-40 \\
-58\end{array}$ & $\begin{array}{l}-44 \\
-38 \\
-11\end{array}$ & $\begin{array}{r}14 \\
11 \\
5\end{array}$ & & $\begin{array}{l}4.94 \\
5.09 \\
4.25\end{array}$ & & & & & & \\
\hline Right inferior temporal gyrus & 52 & -60 & -3 & 92 & 4.24 & & & & & & \\
\hline $\begin{array}{l}\text { Right lateral occipital cortex } \\
\text { Left lateral occipital cortex }\end{array}$ & $\begin{array}{r}25 \\
-41\end{array}$ & $\begin{array}{l}-84 \\
-66\end{array}$ & $\begin{array}{l}3 \\
0\end{array}$ & $\begin{array}{l}54 \\
96\end{array}$ & $\begin{array}{l}4.4 \\
4.91\end{array}$ & Right lateral occipital cortex & 40 & -63 & 3 & 45 & 3.99 \\
\hline $\begin{array}{l}\text { SEM }>\text { SYN } \\
\text { Left anterior inferior frontal gyrus }\end{array}$ & -50 & 30 & 15 & 69 & 4.11 & $\begin{array}{l}\text { SEM }>\text { SYN } \\
\text { Left anterior frontal gyrus }\end{array}$ & $\begin{array}{l}-32 \\
-46\end{array}$ & $\begin{array}{l}24 \\
30\end{array}$ & $\begin{array}{r}-6 \\
11\end{array}$ & 61 & $\begin{array}{l}3.58 \\
3.32\end{array}$ \\
\hline
\end{tabular}

Regions of activation within each native (L1) and non-native (L2) groups for the direct contrasts between response elicited by syntactically anomalous (SYN) vs. semantically anomalous (SEM) sentences $(P<0.05$, corrected). Listed are locations, Talairach coordinates, cluster extents, and maximum $Z$ values. In the left panel, regions for L1 speakers are listed, in the right panel, for L2 speakers. 

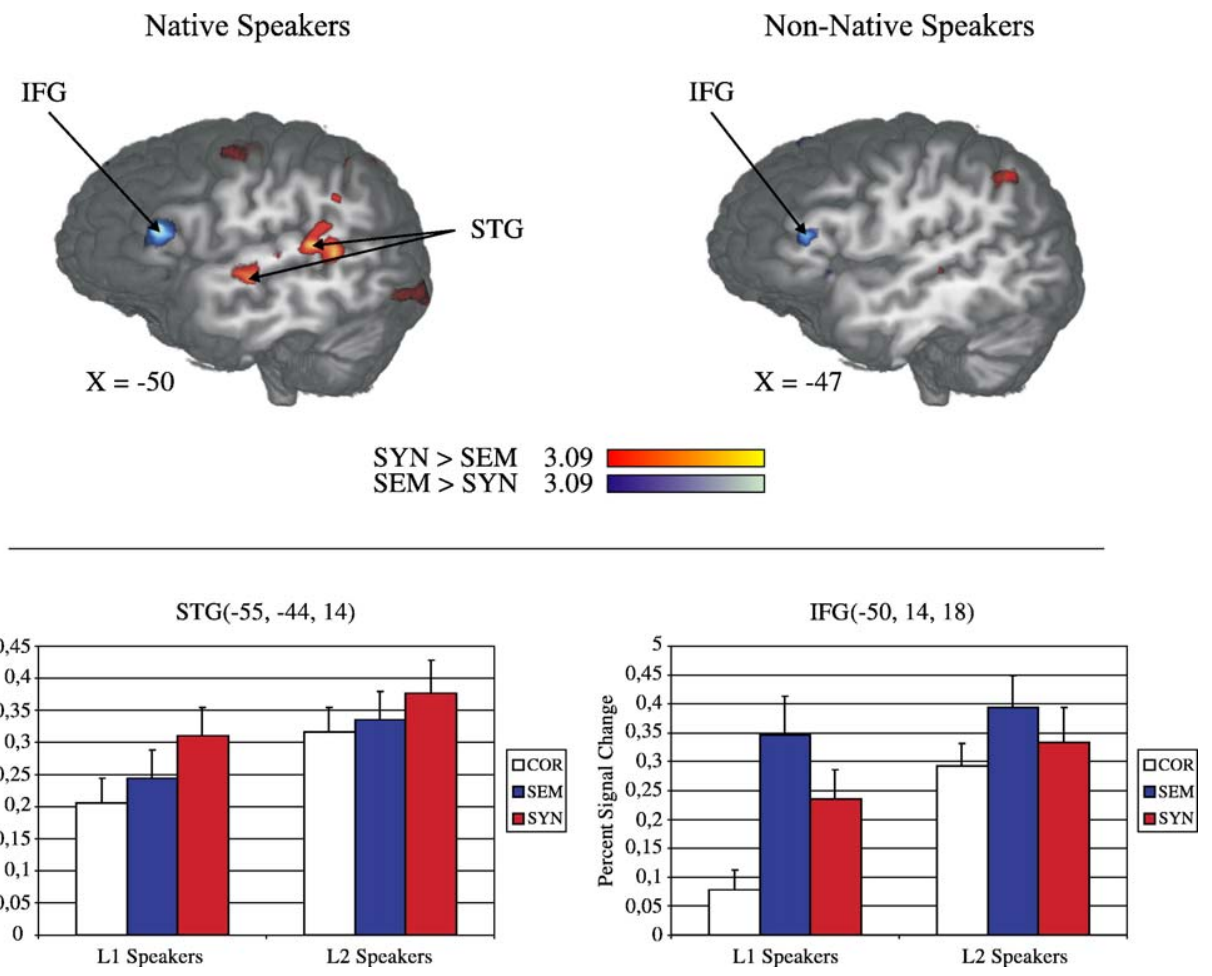

Fig. 3. Direct contrasts between processing of syntactic anomalies (SYN) vs. semantic anomalies (SEM) within the native (L1) and non-native (L2) groups ( $Z>$ 3.09). L1 speakers show greater levels of activation for syntactic processing in superior temporal gyrus (STG) and increased levels of activation for semantic processing in inferior frontal gyrus (IFG). L2 speakers show no differential activation in STG for the processing of the different sentence types, however, more activation is seen in IFG for the processing of SEM. In the bottom panel, mean percent signal change correlated with each sentence condition in the area surrounding the local peak is depicted.

areas to be more active than in L2 speakers. L2 speakers, on the other hand, showed greater levels of activation than L1 speakers in a number of language- and motor-related brain regions.

\section{Differences between groups in language areas}

While processing short, simple, correct sentences, L2 speakers demonstrated greater levels of activation than L1 speakers in several classical language processing areas including left IFG, extending from posterior IFS to anterior IFG (BA45/47) and left MTG.

Both anterior IFG and MTG have been described to support the processing of semantic information (i.e. Rissman et al., 2003). The left IFG, which is discussed in further detail in a following section, is thought to support the strategic retrieval of semantic information and the formation of semantic relationships between lexical items (Thompson-Schill et al., 1997; Wagner et al., 2001). The MTG, on the other hand, has been implicated in supporting multimodal semantic processing in a less specific manner (Booth et al., 2002). Increased activation of MTG has been observed for various semantic tasks in both written (Newmann et al., 2001) and auditory (Rissman et al., 2003) language processing studies. Furthermore, MTG has been shown to be active in a task-dependent (stimulus independent) manner (Friederici et al., 2000b). Specifically, Friederici and colleagues showed that the MTG was more active in participants asked to categorize a word based on semantic information (judgment of abstract/concreteness) than if participants were asked to make a decision based on syntactic information (judgment of word class). Along similar lines, Binder and colleagues argue that MTG and surrounding cortices play an important role in the comprehension of words at a linguisticsemantic level (Binder et al., 1997, 2003).

In the current study, L2 speakers showed increased levels of activation in both IFG and MTG-areas commonly associated with language processing and specifically associated with semantic processing. We suggest that higher activation of a general language processing network in non-native as opposed to native speakers reflects overall higher processing demands for comprehending a foreign language. Furthermore, the greater L2 activation of a network thought to underlie semantic processing in particular suggests that L2 speakers may indeed compensate for uncertainties in language processing by relying on semantic information.

Importantly, no differential activation in left prefrontal or left temporal cortex is observed between L1 and L2 speakers in response to violated sentences. This can be explained by the increased involvement of various portions of the fronto-temporal language network by L1 speakers in response to each anomaly type (see bottom panel of Fig. 3). The difference between groups in response to violation types is therefore less than that seen in response to correct sentences. Put differently, L2 speakers are taxed by the processing of correct sentences in a manner similar to that which we observe in L1 speakers confronted with violated (problematic) language stimuli.

It is interesting to point out that no differences in signal intensity between L1 and L2 participants were observed in extrastriate visual areas, typically considered to be important in identifying and processing orthographic stimuli (Nobre et al., 1994). We had thought that differences in these areas might be 
detected due to the fact that L2 German speakers were native speakers of Russian, a language with a very different orthographic system to that used in German. Therefore, it would not have been surprising to observe an increased involvement of lingual and fusiform gyri associated with a more effortful recognition of orthographic stimuli in the L2 alphabet. The fact that this was not the case, however, suggests that our L2 participants were very adept at reading the German alphabet and that group differences in written language processing occur at levels higher than primary orthographic processing.

\section{Differences between groups in motor preparation network}

L2 speakers showed greater levels of activation in a number of areas generally ascribed to preparation and planning of motor action including bilateral precentral gyrus, preSMA, bilateral postcentral gyrus, extending in the left hemisphere to the angular and supramarginal gyri, as well as structures in the bilateral basal ganglia. It has been shown that reading silently activates premotor and motor areas also involved in overt articulation of words (Hagoort et al., 1999). Such activation has been interpreted previously to reflect articulatory preparation, even in the absence of an overtly articulated task. In the current study, both groups were required to read the experimental stimuli, however, the greater involvement of premotor and motor areas in L2 speakers is suggested to reflect greater difficulties in planning motor control of foreign words. Overt word generation in a foreign language has been shown to engage brain areas responsible for fine motor control previously (Klein et al., 1994, 1995).

Importantly, increased involvement of motor-related areas in L2 speakers is observed in the direct contrast between L2 and L1 speakers while reading sentences in all experimental conditions in our experiment. In other words, these activation differences were present for the processing of language stimuli per se and were not tied to specific difficulties in semantic or syntactic integration.

\section{Within group contrasts: syntactic vs. semantic processing}

Violation paradigms assume that the detection of an error within a specific domain (for example syntax) will cause greater activation of those brain areas responsible for the processing of information in this domain under normal circumstances. In the current study, brain areas selectively responsive to the processing of syntactic and semantic information were captured by directly comparing the brain's response to syntactic anomalies vs. the response to semantic anomalies. By comparing changes in the BOLD response elicited by each of the violation types directly, those portions of the response reflecting general error-detection were eliminated.

\section{L1 speakers}

For the processing of syntactic anomalies, L1 speakers showed a selective increase of activity in left superior temporal cortex, extending from posterior STS to anterior portions of lateral STG. Four local maxima were identified in lateral STG, along an anterior-posterior axis. Different regions within superior temporal cortex have been suggested to support language processing in very specific ways. First of all, secondary and tertiary auditory processing areas in STG/STS, lateral to Heschl's Gyrus, are clearly involved in the processing of speech as a complex acoustic stimulus (Binder et al., 1997; Scott and Johnsrude, 2003).
Secondly, mid to anterior portions of STG have been implicated in the processing of morphosyntactic (Dronkers et al., 2004) as well as syntactic (Friederici et al., 2000a,c, 2003; Newmann et al., 2001; Rüschemeyer et al., 2005) information in spoken sentences. Posterior superior temporal cortex has been suggested to support the sound-based representation of word forms (Scott and Wise, 2004) and the phonological output of speech sounds in the absence of phonological input (Price, 2000) as well as phoneme to grapheme conversion processes (Xu et al., 2001).

While the proximity of STG to primary auditory cortex makes this region an obvious candidate for supporting acoustic processing in general and therefore sound-based language processing, several studies have shown that involvement of superior temporal cortex is not limited to the auditory modality. In one such study, the involvement of classical language areas (including lateral STG) in the processing of both spoken and written sentences was investigated (Michael et al., 2001). Activation in left lateral STG was reported for sentence processing in both modalities - the local maximum of activation in response to written sentences was slightly more posterior than that seen for the processing of spoken sentences. A manipulation of syntactic complexity in written sentences showed a modulation of signal intensity within STG, comparable to that seen in response to acoustically presented sentences, suggesting that the STG supports the processing of syntactic structure regardless of presentation modality. Previous reading studies using both syntactic complexity modulations (Just et al., 1996) and syntactic violations (Embick et al., 2000) also report increased involvement of left lateral STG correlated with increased syntactic processing load. Based on the results of previous sentence processing studies using both spoken and written sentences, we suggest that the extended superior temporal activation observed in L1 speakers reflects syntactic processing on the phrase structure level. Specifically, we suggest that strong preferences for specific word categories based on phrase structure are established by L1 speakers listening or reading sentences. When these expectancies are not fulfilled (template matching), activation in STG is observed. It has been suggested based on electrophysiological studies with patients that posterior portions of the STG may work together with structures of the basal ganglia to support controlled processes of syntactic integration (Friederici and Kotz, 2003).

STG is not, however, the only cortical region implicated repeatedly in the processing of syntactic structure. In fact, a large body of literature, which has attempted to capture on-line syntactic processing by means of syntactic complexity manipulations, has argued that the left frontal cortex (Broca's area) embodies the processing of syntactic structure (review Caplan, 2001). Syntactic complexity has been associated with higher working memory demands due to transformation costs, and it has been suggested that increased activation of IFG in these studies may reflect to some degree the recruitment of short-term memory systems rather than a parsing of syntactic structure (Cooke et al., 2001; Fiebach et al., 2001, 2002). In any case, it seems reasonable to assume that the detection of syntactic violations (as investigated in the current study) may involve processes of a different nature than that captured in complexity manipulations (Embick and Poeppel, 2005; Friderici, 2004).

In addition to left superior temporal cortex, a number of brain regions not thought to reflect specific linguistic processing were observed in conjunction with syntactic anomalies. These included several areas in parietal, occipital and premotor cortices: brain 
regions typically seen in conjunction with the processing of complex visual objects (intraparietal cortex, lateral occipital cortex) as well as motor preparatory regions (right precentral sulcus). Syntactically anomalous sentences were necessarily one word longer than semantically anomalous sentences. We suggest that these activations in parietal, lateral occipital and ventrolateral premotor cortex reflect processing of a greater amount of visual input (additional word) in syntactically anomalous sentences rather than parsing of syntactic structure.

L1 speakers showed a single region of selectively increased activation in response to semantically anomalous sentences. This region of activation was located within left anterior IFG and is very comparable to activation seen in L1 speakers of different languages in response to semantic anomalies in spoken sentences (Rüschemeyer et al., 2005). The involvement of left anterior IFG in processing semantic information is in holding with numerous studies investigating the processing of semantic violations in sentences (Kiehl et al., 2002; Newmann et al., 2001) and specifically strategic retrieval of semantic information (Dapretto and Bookheimer, 1999; Thompson-Schill et al., 1997; Wagner et al., 2001). In particular, the inferior portion of IFG (BA47) has been suggested to play a role in processing semantic relationships between words or phrases or in selecting a word based on semantic features from among competing alternatives (Bookheimer, 2002; Poldrack et al., 1999). In the current study, participants faced with a semantically implausible word in a sentence experience difficulties in establishing a sensible relationship between the anomalous word and the previous sentence context, resulting in increased levels of activation within IFG. Importantly, such activation has nothing to do with long-term storage of semantic representations rather it is thought to reflect a very goal-oriented, strategic process of retrieval (Wagner et al., 2001) or comparison/ analysis (Thompson-Schill et al., 1997). It is only in the realization that a given word does not match the participant's expectations that such IFG activation makes sense in relation to semantic processes.

\section{L2 speakers}

L2 speakers, in contrast to L1 speakers, showed no increased involvement of superior temporal cortex in response to syntactic anomalies. This despite the fact that L2 speakers were quite capable of recognizing syntactically incorrect sentences, as evidenced by their behavioral responses. In a recent fMRI study in which auditory sentence materials were presented to a similar group of L2 speakers, the same null effect within STG in response to syntactic anomalies was observed (Rüschemeyer et al., 2005).

Clearly, a null result is difficult to interpret: it may result from a true lack of difference in signal change intensity between the two conditions in the non-native participants; it may also be the consequence of insufficient power. If the null effect can indeed be taken to reflect no reliable differences between the two experimental conditions, then we can only conjecture that L2 speakers show no greater involvement of STG for processing syntactic than semantic anomalies. This by no means implies that L2 speakers made less use of STG in processing syntactic anomalies - rather it can be said that no difference was detected between the involvement of STG in the processing of syntactically anomalous vs. semantically anomalous sentences (as seen in L1 speakers) in the L2 group. This lack of difference is visualized in the bottom panel of Fig. 3. Here, it is obvious that L2 speakers indeed draw on resources within STG, but to the same degree in all sentence conditions.
L2 speakers did, however, similarly to L1 speakers, show increased levels of activation in several brain regions not typically related directly to language processing. Large portions of right intraparietal cortex as well as right lateral occipital cortex were seen to show more activation for the syntactically anomalous than semantically anomalous sentences. As was stated previously for L1 speakers, these activations are thought to reflect the fact that syntactically anomalous sentences were one word longer than semantically anomalous or correct sentences. Additional reading and processing of additional complex visual objects (i.e. words) are suggested to drive activation in intraparietal and lateral occipital cortices.

L2 speakers showed a comparable pattern of activation to L1 speakers in response to semantic anomalies. Specifically, increased activation was observed in response to semantic anomalies in left anterior IFG. The similarity of response between the two groups is in holding with the results of both a recent fMRI study in which the processing of acoustically presented sentences by L1 and L2 speakers was investigated (Rüschemeyer et al., 2005), as well as the results of electrophysiological studies, which show a reduced but otherwise comparable response of the brain to semantic anomalies in L1 and L2 speakers (Hahne, 2001; Weber-Fox and Neville, 1996). Conceptually, this finding indicates that L2 speakers process lexical-conceptual information of words in sentences in a manner similar to L1 speakers. This extends the increasingly accepted idea of a shared lexical storage system for words in L1 and L2 (for a review, see French and Jacquet, 2004) to a shared lexical access/retrieval system for both systems. Hernandez and colleagues further predict on the basis of a computational model proposed by Li et al. (2004) that overlap of L1 and L2 semantic representations will be greatest for late learners of L2 (Hernandez et al., 2005). Although the authors explicitly state that the dissociation between L1 and L2 semantic representations should exist on a local rather than a global level and may therefore not be detectable by neuroimaging methods such as fMRI, the idea is relevant to the current study.

\section{Modality effects in sentence processing}

Although several studies have been conducted examining modality effects on the processing of single words, fewer studies have directly compared modality effects at the sentence level. In a recent fMRI study, Michael and colleagues asked participants to read and listen to sentences with varying structural complexity and to subsequently judge the validity of a related sentence probe (Michael et al., 2001). The authors investigated changes in the hemodynamic response correlated with the processing of sentence complexity in each of the modalities. As predicted, several brain regions surrounding primary perceptual cortices (i.e. primary auditory and visual cortex) were more active for stimuli presented in the relevant modality. These included extrastriate and parietal cortices for the processing of visual sentence stimuli, and Heschl's gyrus as well as neighboring regions in temporal cortex for the processing of auditory sentence stimuli. In general, however, brain areas associated with higher language processing (for example the left IFG) showed modulation of activity related to sentence complexity regardless of modality. Interestingly, some of these regions showed, in addition to the main effect of complexity, an interaction of modality and complexity. For example, within left IFG, the processing of structurally complex sentences in the auditory modality was seen to elicit a greater change in hemodynamic response than the processing of structurally complex 
sentences in the visual modality. The authors postulate that such differences may reflect the strategies used by readers vs. listeners in dealing with incoming sentence stimuli. Spoken language has a temporal dynamic, which forces the listener to maintain incoming linguistic information for integration. Written language does not maintain this dynamic in the same manner (provided that words are not presented serially in isolation), as the reader can always re-read words in a sentence. The authors suggest, therefore, that the processing of acoustically presented sentences requires participants to make greater use of short-term working memory (and therefore IFG) than does the processing of visually presented sentences. Despite the presence of these interactions within higher cognitive areas, a general overlap of activations was reported for the processing of sentence stimuli in both modalities, suggesting that a language processing network underlies the comprehension of both spoken and written sentences (Michael et al., 2001; see also Constable et al., 2004).

The present study compared the processing of spoken and written sentences in L1 and L2. The results presented here generally support the notion of a common modality-independent processing network, as the results of this reading study were, to a large degree, in agreement with the previously published study in which we investigated spoken language processing (Rüschemeyer et al., 2005). Within group contrasts for L1 and L2 participant, groups for the processing of syntactic and semantic information in both studies were very comparable. In both studies, it is shown in both that processing of syntactic anomalies by L1 speakers elicits a greater signal in mid-STG/STS. In response to auditory presentation, this activation extended to anterior STG, while, in response to visual presentation, this activation extended towards posterior STG/STS. A more anterior focus of STG activation is also reported for spoken vs. written sentences by Michael et al. (2001). The anterior portion of the STG has been shown to come into play, in particular, when syntactic aspects during auditory sentence processing are required (Friederici et al., 2000a). The finding that the anterior STG is involved in on-line syntactic processing in the auditory domain is, moreover, supported by a lesion study indicating that fast syntactic processes are missing in patients with lesions in the anterior portion of the temporal lobe (Friederici and Kotz, 2003) and by an MEG study in which the fast syntactic processes were reflected by two dipoles in the left hemisphere, one of which was located in the anterior STG (Friederici et al., 2000c). The shift of focus within STG to more posterior portions in response to written sentences may reflect slower, more controlled integration processes for written than spoken sentences. Such an interpretation would be supported by work coming from patient studies, showing that lesions in the posterior portion of the temporal lobe hamper late integration (Friederici and Kotz, 2003). It is suggested that the anterior STG supports the establishment of phrase structure expectancies, which are not fulfilled upon encountering a grammatically impossible word in a previously established sentence structure. In neither study do L2 speakers show a differential signal change in STG in response to different correct and incorrect sentence stimuli. Again, this does not reflect a disengagement of STG in L2 speakers, but rather a higher involvement of temporal cortex in response to all sentence conditions, including the incorrect ones (see bottom of Fig. 3). In response to semantic anomalies, however, L1 and L2 participants in both the auditory and the visual study showed increased activation in left IFG. We interpret this finding in light of previous studies to reflect difficulties in conceptually integrating a semantically incongruent word into sentence context.

Despite all the similarities between the two studies, the results of the between group contrasts in the two studies differed slightly from one another with respect to some activations. In the previous study, we reported increased activation for L2 speakers in all language conditions in left IFG, bilateral basal ganglia and the left intraparietal sulcus - which we suggested might reflect additional costs of processing for L2 participants in the phonological domain. In the current study, we again observe increased activation in L2 speakers in left IFG, bilateral basal ganglia and regions surrounding the intraparietal sulcus bilaterally for all sentence conditions. Clearly, L2 speakers in this study were not confronted with having to decipher foreign phonemes in the scanner environment, nevertheless, IFG and basal ganglia activation prevailed. We think that at least some of this activation may reflect increased difficulties in articulatory preparation for L2 speakers reading words in a foreign language silently (an activity which demands covert articulation). In this manner, the coinciding results obtained from stimulus presentation in two different modalities can be somewhat unified.

What escaped observation in the previous (auditory) study was any additional involvement of temporal cortices for L2 speakers. In fact, contrary to the current (visual) study, we observed increased activation in mid-STG for L1 rather than L2 speakers. Two factors may contribute to this discrepancy: (1) the signal increase in the current study for L2 speakers lies within MTG, while signal increase in the auditory study for L1 speakers was located in STG. Signal intensity in inferior and middle temporal areas was not recorded in the auditory experiment. We measured only eight slices in order to reduce the noise created by gradient changes in the scanner as much as possible. Therefore, differences in the response of MTG (as observed in the current experiment) may have simply not been measured. (2) L1 speakers in the auditory study demonstrated greater levels of activation than L2 speakers in and around primary and secondary auditory cortices within the STG/ STS. We interpreted this finding previously to reflect the superior recognition and categorization of phonemes by L1 speakers as compared to L2 speakers. In the current study, participants were not required to decipher acoustic stimuli. Therefore, the increased activation in STG in the auditory study is suggested to be modalityspecific, supporting the efficient processing of phonemes by L1 speakers in regions proximal to primary auditory cortex.

\section{Conclusion}

The processing of different types of linguistic information (syntactic structure and semantic content) by L1 and highly proficient L2 speakers of a language was investigated in a functional magnetic resonance imaging (fMRI) study. A violation paradigm was used, in which syntactically or semantically anomalous as well as correct sentences were presented visually in a random effects design.

In a direct comparison of brain activation between the two groups of participants, L2 speakers showed greater levels of activation than L1 speakers in response to language processing per se in a classical language network comprising: (1) left IFG, extending from posterior IFS to anterior IFG (BA45/47), and (2) left temporal cortex in anterior STG and MTG. Greater role of IFG and MTG is suggested to reflect a greater reliance on semantic 
processing for L2 speakers than L1 speakers. Additionally, L2 speakers showed increased levels of activation in motor-related areas such as preSMA, bilateral structures within the basal ganglia, bilateral precentral gyrus, and bilateral postcentral gyrus extending into supramarginal gyrus and angular gyrus in the left hemisphere. Greater involvement of motor cortices in the reading of a foreign language may reflect greater difficulties for L2 speakers in even covertly reading sentences in L2 (cost of foreign articulationeven if articulation is covert).

Participants in both groups showed selective responses to the processing of the different violation types. For the processing of syntactic anomalies, L1 speakers showed increased involvement in left posterior superior temporal cortex, extending from left STS to mid portions of lateral STG. For the processing of semantic anomalies, L1 speakers showed increased involvement of left anterior IFG. L2 speakers also showed selective responses to syntactic and semantic violations in sentence reading. For the processing of syntactic anomalies, L2 speakers, unlike L1 speakers, did not show an increased involvement of left superior temporal cortex. This was due to a general increase of STG involvement in all sentence conditions. For the processing semantic anomalies, L2 speakers, similar to L1 speakers, showed selective increase in activation in left anterior IFG.

The results of this visual language study were lastly compared to the results of a previously published auditory language study, in which identical sentence stimuli were used. The two sets of results are in general agreement with one another, although brain regions proximal to primary and secondary auditory and visual cortices clearly respond to the presentation modality and the strengths and weakness of each participant group in perceiving language stimuli in a given modality. For example, in response to spoken sentences, L1 speakers showed more activation than L2 participants in STG, which may reflect a greater reliance of L1 speakers on acoustic information in processing language, based on a superior perception of phonemes.

In general, our results show that the differences observed between L1 and L2 speakers reflect greater involvement of specific portions of an overall comparable language network. In other words, participants in both groups show activation of a classical language network, with accentuation in different portions of the network, depending on task demand. We see no indication in this fMRI study for use of a fundamentally different network for reading vs. listening to sentences or for processing an L1 vs. an L2. Research based on other methods of investigation (for example patient studies) may uncover differences not detectable using fMRI.

\section{Acknowledgments}

Many thanks to D.Y. von Cramon, C. Preul, K. Müller, A. Hennenlotter, and G. Supp and two anonymous reviewers for support in the preparation of the manuscript, as well as to M. Naumann, S. Wipke, and A. Wiedemann for their technical support.

\section{References}

Abutalebi, J., Cappa, S., Perani, D., 2001. The bilingual brain as revealed by functional neuroimaging. Biling.: Lang. Cogn. 4 (2), 179-190.
Binder, J., Frost, J., Hammeke, T., Cox, R., Rao, S., Preito, T., 1997. Human brain language areas identified by functional magnetic resonance imaging. J. Neurosci. 17 (1), 353-362.

Binder, J., McKiernan, K., Parsons, M., Westbury, C., Possing, E., Kaufman, J., Buchanan, L., 2003. Neural correlates of lexical access during visual word recognition. J. Cogn. Neurosci. 15 (3), 372-393.

Bookheimer, S., 2002. Functional MRI of language: new approaches to understanding the cortical organization of semantic processing. Annu. Rev. Neurosci. 25, 151-188.

Booth, J., Burman, D., Meyer, J., Gitelman, D., Parrish, T., Mesulam, M.M., 2002. Modality independence of word comprehension. Hum. Brain Mapp. 16, 251-261.

Caplan, D., 2001. Functional neuroimaging studies of syntactic processing. J. Psycholinguist. Res. 30 (3), 297-320.

Chee, M., O’Craven, K., Bergida, R., Rosen, B., Savoy, R., 1999a. Auditory and visual word processing studied with fMRI. HBM 7, 15-28.

Chee, M., Tan, E., Thiel, T., 1999b. Mandarin and English single word processing studied with functional magnetic resonance imaging. J. Neurosci. 19 (8), 3050-3056.

Chee, M., Weekes, B., Lee, K., Soon, C., Schreiber, A., Hoon, J., Chee, M., 2000. Overlap and dissociation of semantic processing of Chinese characters, English words and pictures: evidence from fMRI. NeuroImage 12, 392-403.

Chee, M., Soon, C., Lee, H., 2003. Common and segregated neuronal networks for different languages revealed using functional magnetic resonance adaptation. J. Cogn. Neurosci. 15 (1), 85-97.

Constable, R., Pugh, K., Berroya, E., Mencl, W., Westerveld, M., Ni, W., Shankweiler, D., 2004. Sentence complexity and input modality effects in sentence comprehension: an fMRI study. NeuroImage 22, 11-21.

Cooke, A., Zrif, E., DeVita, C., Alsop, D., Koenig, P., Detre, J., Gee, J., Pinango, M., Balogh, J., Grossman, M., 2001. Neural basis for sentence comprehension: grammatical and short-term memory components. Hum. Brain Mapp. 15, 80-94.

Dapretto, M., Bookheimer, S., 1999. Form and content: dissociating syntax and semantics in sentence comprehension. Neuron 24, 427-432.

Dehaene, S., Dupoux, E., Mehler, J., Cohen, L., Paulesu, E., Perani, D., van de Moortele, P., Lehericy, S., LeBihan, D., 1997. Anatomical variability in the cortical representation of first and second language. NeuroReport 8, 3809-3815.

Dronkers, N., Wilkins, D., Van Valin, R., Redfern, B., Jaeger, J., 2004. Lesion analysis of the brain areas involved in language comprehension. Cognition 92, 145-177.

Embick, D., Poeppel, D., 2005. Mapping syntax using imaging: problems and prospects for the study of neurolinguistic computation. In: Brown, K. (Ed.), Encyclopedia of Language and Linguistics, 2nd ed. Elsevier, Oxford.

Embick, D., Marantz, A., Miyashita, Y., O'Neil, W., Sakai, K., 2000. A syntactic specialization for Broca's areas. Proc. Natl. Acad. Sci. U. S. A. 97, 6150-6154.

Fiebach, C., Schlesewsky, M., Friederici, A., 2001. Syntactic working memory and the establishment of filler-gap dependencies: insights from ERPs and fMRI. J. Psycholinguist. Res. 30, 321-338.

Fiebach, C., Schlesewsky, M., Friederici, A., 2002. Separating syntactic memory costs and syntactic integration costs during parsing: the processing of German WH-questions. J. Mem. Lang. 45, 250-272.

Forman, S., Cohen, J., Fitzgerald, M., Eddy, W., Mintun, M., Noll, D., 1995. Improved assessment of significant activation in functional magnetic resonance imaging (fMRI): use of a cluster-size threshold. MRM 33, 636-647.

French, R., Jacquet, M., 2004. Understanding bilingual memory: models and data. Trends Cogn. Sci. 8 (2), 87-93.

Friderici, A., 2004. Processing local transitions versus long-distance syntactic hierarchies. Trends Cogn. Sci. 8 (5), 245-247.

Friederici, A., 2002. Towards a neural basis of auditory sentence processing. Trends Cogn. Sci. 6 (2), $78-84$.

Friederici, A., Kotz, S.A., 2003. The brain basis of syntactic processes: functional imaging and lesion studies. NeuroImage 20, S8-S17. 
Friederici, A., Meyer, M., von Cramon, D., 2000a. Auditory language comprehension: an event-related fMRI study on the processing of syntactic and lexical information. Brain Lang. 74, 289-300.

Friederici, A., Opitz, B., von Cramon, D.Y., 2000b. Segregating semantic and syntactic aspects of processing in the human brain: an fMRI investigation of different word types. Cereb. Cortex 10, 698-705.

Friederici, A., Wang, Y., Herrmann, C., Maess, B., Oertel, U., 2000c. Localization of early syntactic processes in frontal and temporal cortical areas: a magnetoencephalographic study. Hum. Brain Mapp. 11, 1-11.

Friederici, A., Rüschemeyer, S.-A., Hahne, A., Fiebach, C., 2003. The role of left inferior frontal and superior temporal cortex in sentence comprehension. Cereb. Cortex 13, 170-177.

Friston, K., Fletcher, P., Josephs, O., Holmes, A., Rugg, M., Turner, R., 1998. Event-related fMRI: characterizing differential responses. NeuroImage 7, 30-40.

Hagoort, P., Indefrey, P., Brown, C., Herzog, H., Steinmetz, H., Seitz, R., 1999. The neural circuitry involved in the reading of German words and pseudowords: a PET study. J. Cogn. Neurosci. 11 (4), 383-398.

Hahne, A., 2001. What's different in second-language processing? Evidence from event-related brain potentials. J. Psycholinguist. Res. 30 (3), 251-265.

Hernandez, A., Li, P., MacWhinney, B., 2005. The emergence of competing modules in bilingualism. Trends Cogn. Sci. 9 (5), 220-225.

Illes, J., Francis, W., Desmond, J., Gabrieli, J., Glover, G., Poldrack, R., Lee, C., Wagner, A., 1999. Convergent cortical representation of semantic processing in bilinguals. Brain Lang. 70, 347-363.

Josephs, O., Turner, R., Friston, K., 1997. Event-related fMRI. Hum. Brain Mapp. 5, 243-248.

Just, M., Carpenter, P., Keller, T., Eddy, W., Thulborn, K., 1996. Brain activation modulated by sentence comprehension. Science 274, $114-116$.

Kiehl, K., Laurens, K., Liddle, P., 2002. Reading anomalous sentences: an event-related fMRI study of semantic processing. NeuroImage 17, $842-850$.

Kim, K., Relkin, N., Lee, K., Hirsch, J., 1997. Distinct cortical areas associated with native and second languages. Nature 388, 171-174.

Klein, D., 2003. A positron emission tomography study of presurgical language mapping in a bilingual patient with a left posterior temporal cavernous angioma. J. Neurolinguist. 16, 417-427.

Klein, D., Zatorre, R., Milner, B., Meyer, E., Evans, A., 1994. Left putaminal activation when speaking a second language: evidence from PET. NeuroReport 5, 2295-2297.

Klein, D., Milner, B., Zatorre, R., Meyer, E., Evans, A., 1995. The neural substrates underlying word generation: a bilingual functional-imaging study. Proc. Natl. Acad. Sci. U. S. A. 92, 2899-2903.

Lee, J., Garwood, M., Menon, R., Adriany, G., Andersen, P., Truwit, C., Ugurbil, K., 1995. High contrast and fast three dimensional magnetic resonance imaging at high fields. Magn. Reson. Med. 34, 308.

Li, P., Farkas, I., MacWhinney, B., 2004. Early lexical development in a self-organizing neural network. Neural Netw. 17, 1345-1362.

Lohmann, G., Mueller, K., Bosch, V., Mentzel, H., Hessler, S., Chen, L., Zysset, S., von Cramon, D., 2001. Lipsia-A new software system for the evaluation of functional magnetic resonance images of the human brain. Comput. Med. Imaging Graph. 25, 449-457.

Luke, K., Liu, H., Wai, Y., Wan, Y., Tan, L., 2002. Functional anatomy of syntactic and semantic processing in language comprehension. Hum. Brain Mapp. 16, 133-145.

Marian, V., Spivey, M., Hirsch, J., 2003. Shared and separate systems in bilingual language processing: converging evidence from eyetracking and brain imaging. Brain Lang. 86 (1), $70-82$.

Michael, E., Keller, T., Carpenter, P., Just, M., 2001. FMRI investigation of sentence comprehension by eye and by ear: modality fingerprints on cognitive processes. Hum. Brain Mapp. 13, 239-252.
Mueller, J., 2005. Electrophysiological correlates of second language processing. Second Lang. Res. 21 (2), 152-174.

Newmann, A., Pancheva, R., Ozawa, K., Neville, H., Ullman, M., 2001. An event-related fMRI study of syntactic and semantic violations. J. Psycholinguist. Res. 30 (3), 339-364.

Nobre, A., Allison, T., McCarthy, G., 1994. Word recognition in the human inferior temporal lobe. Nature 372, 260-263.

Norris, D., 2000. Reduced power multi-slice MDEFT imaging. J. Magn. Reson. Imaging 11, 445-451.

Perani, D., Abutalebi, J., 2005. The neural basis of first and second language processing. Curr. Opin. Neurobiol. 15, 202-206.

Perani, D., Dehaene, S., Grassi, F., Cohen, L., Cappa, S., Dupoux, E., Fazio, F., Mehler, J., 1996. Brain processing of native and foreign languages. NeuroReport 7, 2439-2444.

Perani, D., Paulesu, E., Galles, N., Dupoux, E., Dehaene, S., Bettinardi, V., Cappa, S., Fazio, F., Mehler, J., 1998. The bilingual brain: proficiency and age of acquisition of the second language. Brain 121, 1841-1852.

Perani, D., Abutalebi, J., Paulesu, E., Brambati, S., Scifo, P., Cappa, S., Fazio, F., 2003. The role of age of acquisition and language usage in early, high-proficient bilinguals: an fMRI study during verbal fluency. Hum. Brain Mapp. 19, 170-182.

Poldrack, R., Wagner, A., Prull, M., Desmond, J., Glover, G., Gabrieli, J., 1999. Functional specialization for semantic and phonological processing in the left inferior prefrontal cortex. NeuroImage 10, 15-35.

Price, C., 2000. The anatomy of language: contributions from functional neuroimaging. J. Anat. 197, 335-359.

Rissman, J., Eliassen, J., Blumstein, S., 2003. An event-related fMRI investigation of implicit semantic priming. J. Cogn. Neurosci. 15 (8), $1160-1175$.

Rüschemeyer, S.-A., Fiebach, C., Kempe, V., Friederici, A., 2005. Processing lexical semantic and syntactic information in first and second language: fMRI evidence from Russian and German. Hum. Brain Mapp. 25, 266-286.

Scott, S., Johnsrude, I., 2003. The neuroanatomical and functional organization of speech production. Trends Neurosci. 26 (2), 100-107.

Scott, S., Wise, R., 2004. The functional neuroanatomy of prelexical processing in speech production. Cognition 92, 13-45.

Tan, L., Spinks, J., Feng, C., Siok, W., Perfetti, C., Xiong, J., Fox, P., Gao, J., 2003. Neural systems of second language reading are shaped by native language. Hum. Brain Mapp. 18, 158-166.

Thiron, J., 1998. Image matching as a diffusion process: an analogy with Maxwell's demons. Med. Image Anal. 3 (2), 243-260.

Thompson-Schill, S., D’Esposito, M., Aguirre, G., Farah, M., 1997. Role of left inferior prefrontal cortex in retrieval of semantic knowledge: a reevaluation. Proc. Natl. Acad. Sci. U. S. A. 94, 14792-14797.

Ullman, M., 2004. Contributions of memory circuits to language: the declarative/procedural model. Cognition 92, 231-270.

Wagner, A., Pare-Blagoev, E., Clark, J., Poldrack, R., 2001. Recovering meaning: left prefrontal cortex guides controlled semantic retrieval. Neuron 31, 329-338.

Wartenburger, I., Heekeren, H., Abutalebi, J., Cappa, S., Villringer, A., Perani, D., 2003. Early setting of grammatical processing in the bilingual brain. Neuron 37, 159-170.

Weber-Fox, C., Neville, H., 1996. Maturational constraints on functional specializations for language processing: ERP and behavioral evidence in bilingual speakers. J. Cogn. Neurosci. 8, 231-256.

Worsley, K., Friston, K., 1995. Analysis of fMRI time-series revisitedagain. NeuroImage 2, 173-181.

Xu, B., Grafman, J., Gaillard, W., Ishii, K., Vega-Bermudez, F., Pietrini, P., Reeves-Tyler, P., DiCamillo, P., Theodore, W., 2001. Conjoint and extended neural networks for the computation of speech codes: the neural basis of selective impairment in reading words and pseudowords. Cereb. Cortex 11 (3), 267-277. 